\title{
Multimodal Affect and Aesthetic Experience
}

\author{
Theodoros Kostoulas \\ Department of Computing and Informatics \\ Bournemouth University, UK \\ tkostoulas@bournemouth.ac.uk \\ Theodora Chaspari \\ Computer Science \& Engineering Department \\ Texas A\&M University, USA \\ chaspari@tamu.edu
}

\author{
Michal Muszynski \\ University of Geneva \& Carnegie Mellon University \\ Switzerland and USA \\ Michal.Muszynski@unige.ch \\ Panos Amelidis \\ Creative Technology Department \\ Bournemouth University, UK \\ pamelidis@bournemouth.ac.uk
}

\begin{abstract}
The term "aesthetic experience" corresponds to the inner state of a person exposed to form and content of artistic objects. Exploring certain aesthetic values of artistic objects, as well as interpreting the aesthetic experience of people when exposed to art can contribute towards understanding (a) art and (b) people's affective reactions to artwork. Focusing on different types of artistic content, such as movies, music, urban art and other artwork, the goal of this workshop is to enhance the interdisciplinary collaboration between affective computing and aesthetics researchers.
\end{abstract}

\section{CCS CONCEPTS}

- Computing methodologies $\sim$ Artificial intelligence

\section{KEYWORDS}

Affective computing, Aesthetic experience, Multimodal modeling, Signal processing, Machine Learning, Emotions

\section{ACM Reference format:}

Theodoros Kostoulas, Michal Muszynski, Theodora Chaspari and Panos Amelidis. 2020. Multimodal Affect and Aesthetic Experience. In Proceedings of 2020 ACM Conference on Multimodal Interaction (ICMI '20), October 25-29, 2020, Utrecht, the Netherlands. ACM, New York, NY, USA, 2 pages. https://doi.org/10.1145/3382507.3420055

\section{Introduction}

Aesthetic experience corresponds to the personal experience when engaged with art and differs from the everyday experience

Permission to make digital or hard copies of all or part of this work for personal or classroom use is granted without fee provided that copies are not made or distributed for profit or commercial advantage and that copies bear this notice and the full citation on the first page. Copyrights for components of this work owned by others than ACM must be honored. Abstracting with credit is permitted. To copy otherwise, or republish, to post on servers or to redistribute to lists, requires prior specific permission and/or a fee. Request permissions from Permissions@acm.org.

ICMI '20, October 25-29, 2020, Virtual Event, Netherlands.

(c) 2020 Association of Computing Machinery.

ACM ISBN 978-1-4503-7581-8/20/10...\$15.00.

DOI: https://doi.org/10.1145/3382507.3420055. which deals with the interpretation of natural objects, events, environments and people $[3,13]$. The exploration of aesthetic emotions, i.e. the emotions experienced during an aesthetic experience, can provide the means for better understanding why humans choose to make and engage with art and the features of artistic objects that affect our experience.

Within attempts to understand art, both past and recent research efforts had focused on exploring accounts given by writers, artists and philosophers as well as on exploring debates about empathy in aesthetic experience [1,11,12]. Similarly, recent work has explored the interaction of the human with the urban environment $[5,6,20]$. Depending on its shape and structure, the urban environment can serve as a significant factor for promoting human content, but also contributing to its decline. Measuring multimodal affective reactions would provide the means for better understanding emotions elicited from objects or moments of aesthetic value [14].

In the meantime, measuring and processing multimodal affective reactions includes addressing several challenges, such as how to synchronously capture signals of various natures (physiological and behavioral), of different spatial and temporal dimensions. Dealing with these challenges would enable robust affect modelling utilizing multimodal signals and would provide the means for better interpretation of the cognitive structure of affective states $[10,17,18]$ and affective reactions during aesthetic experience $[9,16,19]$.

The goal of this workshop is to connect researchers and advance the state of the art in the affective computing and aesthetics research areas. In this sense, this workshop builds on existing work, also, from its organisers [2,4,5,7,8,15].

The following topics are addressed:

- Multimodal data collection in response to artistic objects

- Emotions and aesthetic experience

- Qualitative and quantitative evaluation of the experience and absorption of aesthetic values

- Analysis of physiological and behavioral signals captured during aesthetic experience

- Assessment of affective states in response to movies, music, and other artwork 
- Content analysis of artistic objects (movies, music, paintings, games, etc.)

- Intrapersonal and interpersonal correlation of multimodal responses during aesthetic experience

- Neuroaesthetics for studying the underlaying brain mechanisms and sensory, reward, emotional neural processes related to aesthetics

- Human-habitat interaction and its relation to human content and well-being

\section{Expected contributions and impact}

In the affective computing research field, contributions from this workshop will help advancing the state-of-the-art with respect to the multimodal exploration of the inner state of a person involved in art, analysis of multimodal reactions to artistic content, intrapersonal and interpersonal synchrony of multimodal responses. From the artistic studies point of view, this workshop will contribute towards understanding the adequacy of the intuitions of critics, filmmakers and subjective judgements with the emotions elicited, as well as examining whether the judgment of an aesthetic value of an object is distinguishable from the aesthetic experience and the associated aesthetic emotions.

Industries such as media and advertisement companies are keen to explore new methods for analyzing, comprehending and predicting spectators' reactions to films, clips or music pieces, thus they would be interested in the findings presented in this workshop. Finally, other related fields can benefit from the research efforts presented in this workshop, such as HCI, social computing, gaming, etc.

\section{Keynote and mini presentations}

The keynote will be delivered by Dr. Mohammad Soleymani, research assistant professor with the USC Institute for Creative Technologies. The keynote is entitled "Machine understanding of emotion and sentiment". Mini presentations will also be delivered covering the topics of the workshops, including: "affective computing and the built environment" and "affective computing, aesthetic and persuasive experiences".

\section{ACKNOWLEDGMENTS}

The organizers of the workshop would like to thank the workshop committee members: Guillaume Chanel (University of Geneva), Christos Iliou (Information Technologies Institute, CERTH), Iosif Mporas (University of Hertfordshire), Constantinos Marios Angelopoulos (Bournemouth University), Akane Sano (Rice University).

\section{REFERENCES}

[1] André Bazin. 1967. What Is Cinema? Hugh Gray (Berkeley, CA: University of California Press, 1967): 14.

[2] Theodora Chaspari, Andreas Tsiartas, Leah I. Stein, Sharon A. Cermak, and
Shrikanth S. Narayanan. 2015. Sparse Representation of Electrodermal Activity With Knowledge-Driven Dictionaries. IEEE Transactions on

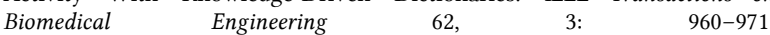
https://doi.org/10.1109/TBME.2014.2376960

[3] Gerald C Cupchik, Oshin Vartanian, Adrian Crawley, and David J Mikulis. 2009. Viewing artworks: contributions of cognitive control and perceptual facilitation to aesthetic experience. Brain and cognition 70, 1: 84-91.

[4] Connor Fletcher, Vedad Hulusic, and Panos Amelidis. 2019. Virtual reality ear training system: A study on spatialised audio in interval recognition. In 2019 11th International Conference on Virtual Worlds and Games for Serious Applications, VS-Games $2019 \quad$ - $\quad$ Proceedings, $1 D U U M Y$. https://doi.org/10.1109/VS-Games.2019.8864592

[5] Jinwoo Kim, Megha Yadav, Changbum R. Ahn, and Theodora Chaspari. 2019 Saliency Detection Analysis of Pedestrians' Physiological Responses to Assess Adverse Built Environment Features. In Computing in Civil Engineering 2019: Smart Cities, Sustainability, and Resilience - Selected Papers from the ASCE International Conference on Computing in Civil Engineering 2019, 90-97. https://doi.org/10.1061/9780784482445.012

[6] Jinwoo Kim, Megha Yadav, Theodora Chaspari, and Changbum R. Ahn. 2020 Saliency detection analysis of collective physiological responses of pedestrians to evaluate neighborhood built environments. Advanced Engineering Informatics 43: 101035. https://doi.org/10.1016/j.aei.2020.101035

[7] Theodoros Kostoulas, Guillaume Chanel, Michal Muszynski, Patrizia Lombardo, and Thierry Pun. 2015. Dynamic Time Warping of Multimodal Signals for Detecting Highlights in Movies. In Proceedings of the 1st Workshop on Modeling INTERPERsonal SynchrONy And infLuence, ICMI 2015, 35-40.

[8] Theodoros Kostoulas, Guillaume Chanel, Michal Muszynski, Patrizia Lombardo, and Thierry Pun. 2015. Identifying aesthetic highlights in movies from clustering of physiological and behavioral signals. In Quality of Multimedia Experience (QoMEX), 2015 Seventh International Workshop on, IEEE.

[9] Theodoros Kostoulas, Guillaume Chanel, Michal Muszynski, Patrizia Lombardo, and Thierry Pun. 2017. Films, Affective Computing and Aesthetic Experience: Identifying Emotional and Aesthetic Highlights from Multimodal Signals in a Social Setting. Frontiers in ICT 4: 11 https://doi.org/10.3389/fict.2017.00011

[10] Eleni Kroupi, J-M Vesin, and Touradj Ebrahimi. 2013. Phase-Amplitude Coupling between EEG and EDA while experiencing multimedia content. In Affective Computing and Intelligent Interaction (ACII), 2013 Humaine Association Conference on, 865-870.

[11] Patrizia Lombardo. 2011. \{É\}motion et souvenir chez Aldo Rossi. Faces-fournal d'Architecture, 69: 34.

[12] Patrizia Lombardo. 2014. Literature, Emotions, and the Possible: Hazlitt and Stendhal. In Mind, Values, and Metaphysics. Springer, 117-134.

[13] Slobodan Marković. 2012. Components of aesthetic experience: aesthetic fascination, aesthetic appraisal, and aesthetic emotion. i-Perception 3, 1: 1-17.

[14] Michal Muszynski. 2018. Recognizing film aesthetics, spectators\&\#039; affect and aesthetic emotions from multimodal signals. https://doi.org/10.13097/ARCHIVE-OUVERTE/UNIGE:114609

[15] Michal Muszynski, Theodoros Kostoulas, Guillaume Chanel, Patrizia Lombardo, and Thierry Pun. 2015. Spectators' Synchronization Detection based on Manifold Representation of Physiological Signals: Application to Movie Highlights Detection. In Proceedings of the 2015 ACM on International Conference on Multimodal Interaction, 235-238.

[16] Michal Muszynski, Leimin Tian, Catherine Lai, Johanna Moore, Theodoros Kostoulas, Patrizia Lombardo, Thierry Pun, and Guillaume Chanel. 2019 Recognizing Induced Emotions of Movie Audiences From Multimodal Information. IEEE Transactions on Affective Computing: 1-1. https://doi.org/10.1109/taffc.2019.2902091

[17] Andrew Ortony, Gerald L Clore, and Allan Collins. 1990. The cognitive structure of emotions.

[18] Klaus R Scherer. 2003. Introduction: Cognitive components of emotion

[19] Leimin Tian, Michal Muszynski, Catherine Lai, Johanna D. Moore, Theodoros Kostoulas, Patrizia Lombardo, Thierry Pun, and Guillaume Chanel. 2017. Recognizing induced emotions of movie audiences: Are induced and perceived emotions the same? In 2017 Seventh International Conference on Affective Computing and Intelligent Interaction (ACII), 28-35. https://doi.org/10.1109/ACII.2017.8273575

[20] Megha Yadav, Theodora Chaspari, Jinwoo Kim, and Changbum R. Ahn. 2018 Capturing and quantifying emotional distress in the built environment. In Proceedings of the Human-Habitat for Health (H3): Human-Habitat Multimodal Interaction for Promoting Health and Well-Being in the Internet of Things era20th ACM International Conference on Multimodal Interaction, ICMI 2018, 1-8. https://doi.org/10.1145/3279963.3279967 